\title{
Micron-scale organic thin film transistors with conducting polymer electrodes patterned by polymer inking and stamping
}

\author{
Dawen Li and L Jay Guo ${ }^{\text {a) }}$ \\ Department of Electrical Engineering and Computer Science, The University of Michigan, \\ Ann Arbor, Michigan 48109
}

(Received 10 October 2005; accepted 6 December 2005; published online 10 February 2006)

\begin{abstract}
We report organic thin film transistors (OTFTs) with conductive polymer poly (3,4-ethylenedioxythiophene)/poly(4-styrenesulphonate) (PEDOT) electrodes that are fabricated by a simple polymer inking and stamping technique. An OTFT channel length of $2 \mu \mathrm{m}$ has been achieved. This patterning technique is a purely additive process, which does not affect the functionality of the conductive polymers, and is fully compatible for patterning on a flexible substrate. Electrical characteristics of top contact (TC) pentacene TFTs with PEDOT electrodes is superior to those with gold electrodes due to a lower carrier injection barrier. Extracted contact resistance shows that the channel length of TC OTFTs can be further reduced to increase the drain current. (C) 2006 American Institute of Physics. [DOI: 10.1063/1.2168669]
\end{abstract}

Organic electronics have promising applications due to two distinct advantages: low cost over a large area and lowtemperature fabrication on a flexible substrate. ${ }^{1}$ The substitution of gold electrodes with a conductive polymer ${ }^{2-4}$ is a significant step forward for reducing material cost and for fabrication on flexible substrates. Moreover, conductive polymer electrodes could provide lower-energy barriers for hole carrier injection. ${ }^{5}$ An effective and scalable patterning technique is critical for the low-cost fabrication of organic electronic devices. Previously investigated techniques, such as screenprinting, ${ }^{6}$ ink-jet printing, ${ }^{7,8}$ and microcontact printing, 9 either have low resolution or involve further process steps, which may degrade the functionality of polymer materials. In contrast, the polymer inking and stamping technique reported in this paper possesses the capability of patterning very high resolution features and is inherently high-throughput. This method directly patterns functional materials on substrates, which does not require additional solvent and plasma processing steps and therefore preserves the functionality of the organic materials.

In this study, a conducting polymer poly(3,4-ethyl enedioxythiophene)/poly(4-styrenesulphonate) (PEDOT/ PSS, Baytron P) was patterned as the source and the drain electrodes by using the polymer inking and stamping technique, with a schematic shown in Fig. 1(a). First conductive polymer PEDOT is spin coated on a patterned polydimethylsiloxane (PDMS) elastomer stamp. A brief $\mathrm{O}_{2}$ plasma treatment of PDMS mold (250 mTorr, $80 \mathrm{~W}, 6-10 \mathrm{~s}$ ) is necessary to create a hydrophilic surface ${ }^{10}$ for spin casting of a PEDOT aqueous solution to form a uniform PEDOT film on the PDMS stamp. An atomic force microscopy (AFM) image [Fig. 1(b)] shows the cross-sectional profile of PEDOT on a PDMS stamp, and reveals that the coated PEDOT at the edge of protrusions is thinner than on the other surfaces of the PDMS stamp. Next, the PDMS stamp with coated PEDOT is placed on a $\mathrm{Si} / \mathrm{SiO}_{2}$ substrate to make a conformal contact. The assembly of the PDMS mold and the substrate is baked on hot plate at $80^{\circ} \mathrm{C}$ for $2 \mathrm{~min}$. Finally, the PDMS stamp is removed from the substrate, leaving

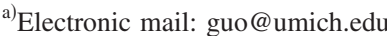

positive PEDOT patterns (i.e., identical to the protrusion features on the stamp) on the substrate. This technique is fully compatible for patterning on flexible substrate since PEDOT is spin coated on PDMS stamp, and the PDMS stamp itself is flexible, which ensures conformal contact with the flexible substrates. The flexible substrates we have experimented include Poly Ether Sulfone (PES), Poly(ethylene Terephthalate) (PET), and poly(vinyl chloride) (PVC) thin films. As an example, Fig. 1(c) shows the circular PEDOT patterns on a flexible substrate with high resolutions. Patterned PEDOT source/drain electrodes for both bottom contact (BC) and TC pentacene TFTs are illustrated in Figs. 1(d) and 1(e), respectively. The smallest gap width (which defines the OTFT channel length) is $2 \mu \mathrm{m}$, limited only by the minimum feature size on the stamp that was used. Surface profile characterization shows that the edge of the transferred PEDOT pattern has a slightly tapered profile.

Successful pattern transfer depends on the substrate surface condition as well as the PEDOT aqueous dispersion. For
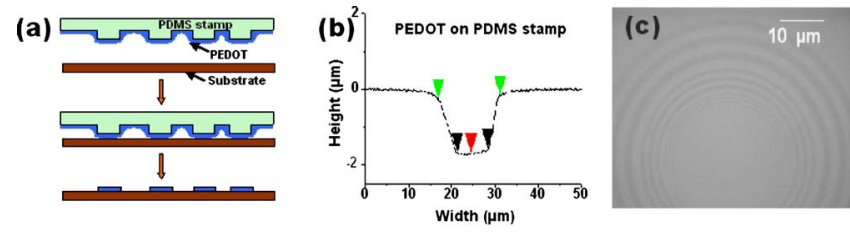

(d)

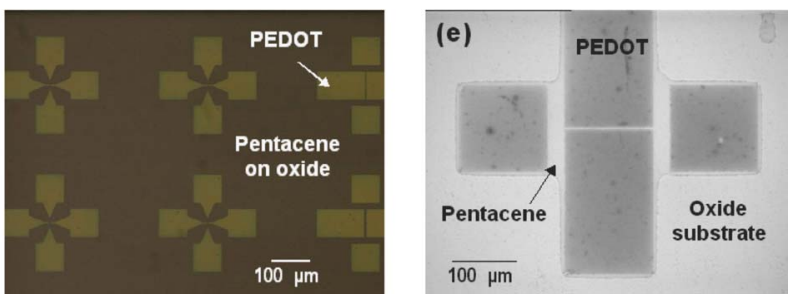

FIG. 1. (Color online) (a) Schematic of polymer inking and stamping process for patterning PEDOT conducting polymer. (b) Cross-sectional AFM image of PEDOT on PDMS stamp (c) Concentric ring patterns transferred on PES flexible substrate. (d) PEDOT electrode patterns transferred on a $\mathrm{SiO}_{2}$ substrate for BC OTFTs, gap width from 2 to $10 \mu \mathrm{m}$. (e) PEDOT electrode patterns transferred on a pentacene thin film surface for TC OTFTs, $W / L=140 \mu \mathrm{m} / 2 \mu \mathrm{m}$. Pentacene outside of the PEDOT electrode pads adhered to the stamp and are removed when the PMDS stamp is peeled off from the substrate. 
the fabrication of BC OTFTs, spin-coated PEDOT is directly transferred onto the substrate. A very small amount of adhesive agent Silquest 187 (Dow Corning) was either added into the PEDOT aqueous dispersion or vapor coated onto the substrate to ensure the adhesion of the PEDOT film. Normally $\mathrm{O}_{2}$ plasma treatment $(250 \mathrm{mTorr}, 80 \mathrm{~W}, 60 \mathrm{~s}$ ) of substrate is carried out before the pattern transfer process to ensure a clean surface and to improve surface hydrophilicity. $\mathrm{O}_{2}$ plasma treatment of substrates $\left(\mathrm{Si} / \mathrm{SiO}_{2}\right.$ substrate or $\mathrm{PVC}$, PET, PES flexible substrates) creates hydroxyl $(-\mathrm{OH})$ groups that can form a siloxane bond with the Silquest molecules. The epoxy end of the Silquest molecules serves as the coupling agent for the PSS in the PEDOT film. When peeling the stamp from the substrate, the highest stress was generated at the edge of the PDMS protrusion patterns. Also, as pointed out earlier, the AFM characterization shows that the PEDOT is thinnest at such edges. When the stress at the edge exceeds the yield stress of the PEDOT material, the thin PEDOT film will break around the protrusion edges and PEDOT patterns can be transferred onto the substrate.

The baking process at $80^{\circ} \mathrm{C}$ prior to stamp/substrate separation serves three purposes. First, it facilitates the chemical bond formation for the Silquest coupling agent with the substrate and the PSS. Second, it helps to accelerate the reversion of $\mathrm{O}_{2}$ plasma-treated PDMS stamp from hydrophilic back to a hydrophobic state. A hydrophobic PDMS surface after spin coating of PEDOT is preferred in order for the conductive polymer to be easily detached from the stamp and transferred onto the substrate. Third, thermal curing and annealing of the PEDOT film stabilizes the defined PEDOT patterns. ${ }^{11}$ For the fabrication of TC OTFTs (source and drain electrodes on top of the organic semiconductor), PEDOT patterns are transferred onto a pentacene layer that is thermally evaporated onto the $\mathrm{Si} / \mathrm{SiO}_{2}$ substrate. Prior to the deposition of a pentacene semiconductor layer, the $\mathrm{SiO}_{2}$ surface was treated with an octadecyltrichlorosilane (OTS) monolayer to increase the adhesion of deposited pentacene on the substrate. OTS treatment also reduces surface energy and improves the ordering of the pentacene film, which leads to increased field-effect mobility and a reduced subthreshold swing. ${ }^{12}$

In this process, a controlled amount of glycerol is added into PEDOT aqueous dispersion for pattern transfer for both $\mathrm{TC}$ and $\mathrm{BC}$ devices. Glycerol acts as a plasticizer, which increases the chain mobility of the polymers, resulting in a lower processing temperature and pressure. ${ }^{13}$ Adding glycerol to the PEDOT solution also prevents the spin-coated PEDOT thin film from completely drying out and makes it stick better to the substrate during the pattern transfer. Moreover, adding glycerol can also enhance the conductivity of PEDOT material ${ }^{14,15}$ PEDOT suspension with glycerol was spin coated at $3 \mathrm{krpm}$ for $30 \mathrm{~s}$, a typical thickness of PEDOT thin film was about $100 \mathrm{~nm}$, with a sheet resistance of $\sim 6 \times 10^{4} \Omega / \square$.

There are two distinctive advantages of using this polymer inking technique to make TC devices. First, the PEDOT electrodes are directly transferred onto the pentacene film, which eliminates any solvent process that may damage the pentacene film or its electrical property. Second, the pentacene in the active device region are defined simultaneously during the PEDOT pattern transfer [Fig. 1(e)]. When the PDMS stamp is placed on the substrate deposited with pentacene, a slight force exerts on the PDMS mold to ensure
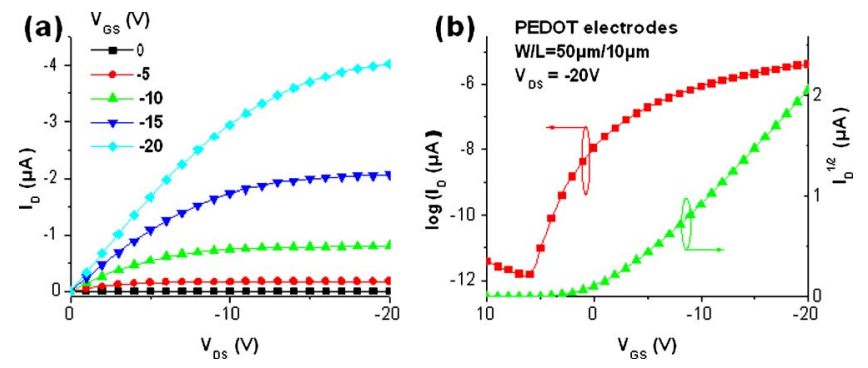

FIG. 2. (Color online) (a) Electrical output characteristics and (b) transfer characteristics of top contact pentacene TFTs $(W / L=5$, channel length of $10 \mu \mathrm{m}$ ) with PEDOT electrodes. Characterization was carried out by applying $V_{\mathrm{DS}}$ and $V_{\mathrm{GS}}$ of less than $-20 \mathrm{~V}$.

conformal contact between the mold and the substrate, which causes the deformation of PDMS mold due to its very low Young's modulus. Recess areas of PDMS stamp contact the pentacene thin film, and remove the pentacene film that surrounds source/drain pads to the PDMS stamp during stamp separation. This feature is very desirable because no further patterning of organic semiconductor active layer ${ }^{16,17}$ is required to create electrical isolation between the devices, which significantly improves the current on/off ratio.

Figures 2(a) and 2(b) show the typical electrical output and transfer characteristics of TC pentacene TFTs with PEDOT electrodes, respectively. For TC pentacene TFTs with a channel length of $25 \mu \mathrm{m}$, the field-effect mobility of $0.71 \mathrm{~cm}^{2} / \mathrm{V} \mathrm{s}$ in the saturation regime $\left(V_{\mathrm{DS}}=-20 \mathrm{~V}\right.$ and $V_{\mathrm{GS}}=-20 \mathrm{~V}$ ), on/off current ratio of $10^{6}$, subthreshold swing of $1 \mathrm{~V} /$ decade, and a threshold voltage of $-2 \mathrm{~V}$ were obtained. Moreover, the electrical performance of pentacene TFTs with PEDOT electrodes showed better characteristics than those fabricated using gold electrodes for TC OTFT configurations. TC OTFTs with gold electrodes $(W / L=20$, channel length of $200 \mu \mathrm{m}$ from a shadow mask), serving as a reference device was fabricated during the same pentacene vacuum deposition. The pentacene thin film of $40 \mathrm{~nm}$ thickness was thermally evaporated in a high vacuum (below 5 $\times 10^{-6}$ Torr) with a substrate temperature of $25^{\circ} \mathrm{C}$, and a deposition rate of $0.5 \AA / \mathrm{s}$. The field-effect mobility of $0.15 \mathrm{~cm}^{2} / \mathrm{V} \mathrm{s}$, the on/off current ratio on the order of $10^{4}$, subthreshold swing of $2.8 \mathrm{~V} /$ decade, and the threshold voltage of $-5 \mathrm{~V}$ were obtained for the Au contact reference device. The significantly improved performance for the PEDOT contact OTFT can be understood by comparing the carrier injection barrier heights. Carrier injection energy barriers between pentacene and electrodes (both gold and PEDOT) have been investigated previously by using ultraviolet photoemission spectroscopy. ${ }_{5,18}$ It was determined that the carrier (hole) injection barrier at the PEDOT/pentacene interface $(0.25 \mathrm{eV})$ is significantly smaller than that of the $\mathrm{Au} /$ pentacene interface $(0.85 \mathrm{eV})$, despite the very similar work functions $(\sim 5 \mathrm{eV})$ of the two materials. For TC device structure, the pentacene thin film is uniform over the whole substrate. Both PEDOT and gold electrode pads have good contact with the pentacene film. The morphology of pentacene thin film is primarily determined by the substrate surface characteristics, and is anticipated to be similar between the two types of devices. In this case, the energy barrier between the electrode and the organic semiconductor pentacene dominates the carrier injection efficiency, and the carrier injection from PEDOT to pentacene is more efficient 

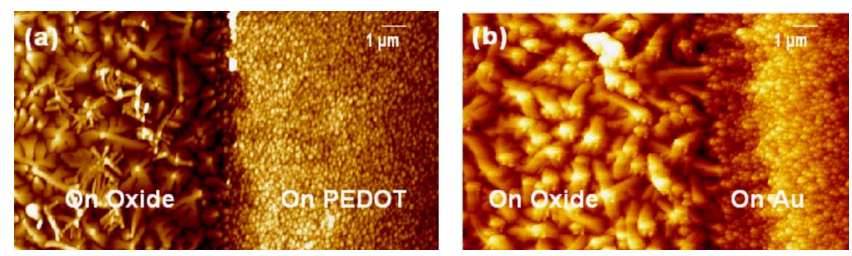

FIG. 3. (Color online) AFM (Digital Instruments, tapping mode) images for (a) pentacene on PEDOT electrodes, and (b) pentacene on gold electrodes. Large grain sizes are pentacenes in the OTFT's channel, and small pentacene grains are on the top and the boundaries of the electrodes.

than from gold for the TC OTFT configuration.

On the other hand, the electrical performance of the $\mathrm{BC}$ OTFTs was very similar between OTFTs with PEDOT electrodes and those with $\mathrm{Ti} / \mathrm{Au}$ electrodes. The field-effect mobility is on the order of $10^{-2} \mathrm{~cm}^{2} / \mathrm{V} \mathrm{s}$, on/off current ratio of $10^{4}-10^{5}$, a subthreshold swing of $2-3 \mathrm{~V} /$ decade, and threshold voltage in the range of -3 to $-5 \mathrm{~V}$. As we know, the carriers injection from electrode into organic semiconductor depends on both the interface morphology and the energy barrier between the electrode and the organic semiconductor. Atomic Force Microscopy (AFM) characterization revealed that the grain sizes of pentacene on PEDOT electrodes and on $\mathrm{Ti} / \mathrm{Au}$ electrodes as well as at the electrode/channel interfaces are much smaller as compared with those in the channel region, i.e., pentacene directly on top of the gate insulator (Fig. 3). Although the energy barrier for carrier injection between the two types of electrodes and the pentacene is different, the very small pentacene grains limit the overall carrier injection from the electrode to the organic semiconductor, leading to similar electrical behavior between the two types of devices.

Another interesting finding is that the extracted mobility from the transfer characteristics for TC OTFTs is in the range of $0.3-0.7 \mathrm{~cm}^{2} / \mathrm{V} \mathrm{s}$, and is strongly dependent on the channel length, as shown in Fig. 4(a). As channel length is reduced, the extracted field-effect mobility decreases. In contrast, the extracted field-effect mobility for BC pentacene TFTs is on the order of $10^{-2} \mathrm{~cm}^{2} / \mathrm{V} \mathrm{s}$, and has less dependence on the channel length. This apparent dependence of mobility on channel length can be explained by taking into account the contact resistance between the pentacene and the PEDOT, which is a series resistance to the intrinsic pentacene channels. The effect of contact resistance on the output current becomes stronger as the channel length becomes smaller, as it is becoming comparable to the channel resistance. Figure 4(b) shows that the contact resistance of TC OTFTs has the same order of magnitude as the channel resistance per micron. As the channel length increases, contact resistance becomes less weighted in the total resistance. Therefore, the extracted field-effect mobility becomes higher when the channel length increases [shown in Fig. 4(a)]. On the other hand, the contact resistance of the BC OTFTs is about two orders of magnitude greater than their channel resistances, scaling, down the channel length will not increase the drain current significantly. The extracted fieldeffect mobility increases very slowly with the channel length because the contact resistance dominates the total resistance in the BC OTFTs. For both TC and BC devices, the series resistance of PEDOT electrodes themselves is less than $1 \%$ of total contact resistance.
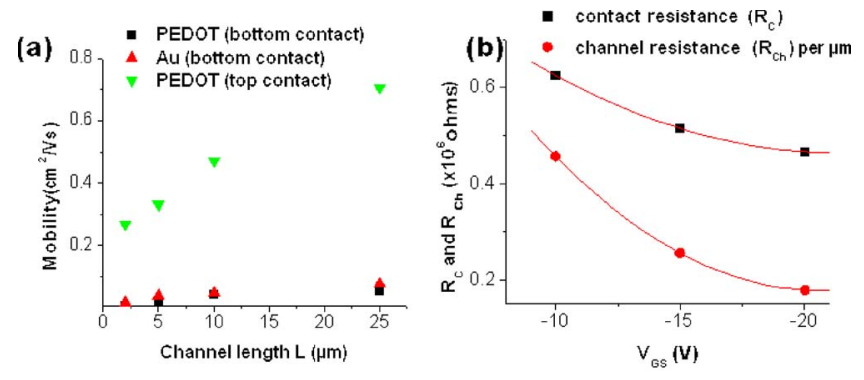

FIG. 4. (Color online) (a) A comparison of the extracted field-effect mobilities with different channel lengths between TC and BC OTFTs. (b) For TC configuration OTFTs with PEDOT electrodes, contact resistance $R_{C}$ is comparable to channel resistance $R_{\mathrm{Ch}}$.

In summary, we developed a polymer inking and stamping technique to pattern OTFTs with conductive polymer PEDOT electrodes. Both TC and BC OTFTs demonstrate excellent electrical characteristics. Field-effect mobility and the on/off current ratio in the saturation regime as large as $0.7 \mathrm{~cm}^{2} / \mathrm{V} \mathrm{s}$ and on the order of $10^{6}$ were obtained. This polymer inking and stamping technique is very suitable for patterning on a flexible substrate, and it can be potentially developed as a reel-to-reel process technology for patterning conductive polymers.

This work was supported by NSF Grant No. ECS 0424204. Special thanks to Dr. Woong Sang Jahng for assisting the OTS treatment of $\mathrm{SiO}_{2}$ and pentacene deposition, and Dr. Hyunsik Moon for pentacene purification.

${ }^{1}$ J. A. Rogers and Z. Bao, J. Polym. Sci., Part A: Polym. Chem. 40, 3327 (2002).

${ }^{2}$ T. Kawase, H. Sirringhaus, R. H. Friend, and T. Shimoda, Adv. Mater. (Weinheim, Ger.) 13, 1601 (2001).

${ }^{3}$ M. Halik, H. Klauk, U. Zschieschang, G. Schmid, W. Radlik, and W. Weber, Adv. Mater. (Weinheim, Ger.) 14, 1717 (2002).

${ }^{4}$ M. Lefenfeld, G. Blanchet, and J. A. Rogers, Adv. Mater. (Weinheim, Ger.) 15, 1188 (2003).

${ }^{5}$ N. Koch, A. Kahn, J. Ghijsen, J.-J. Pireaux, J. Schwartz, R. L. Johnson, and A. Elschner, Appl. Phys. Lett. 82, 70 (2003).

${ }^{6}$ D. A. Pardo, G. E. Jabbour, and N. Peyghambarian, Adv. Mater. (Weinheim, Ger.) 12, 1249 (2000).

${ }^{7}$ H. Kipphan, Handbook of Print Media: Technologies and Production Methods (Springer Verlag, New York, 2001).

${ }^{8}$ H. Sirringhaus, T. Kawase, R. H. Friend, T. Shimoda, M. Inbasekaran, W. Wu, and E. P. Woo, Science 290, 2123 (2000).

${ }^{9}$ K. M. Choi and J. A. Rogers, J. Am. Chem. Soc. 125, 4060 (2003).

${ }^{10}$ Y.-L. Loo, R. L. Willett, K. W. Baldwin, and J. A. Rogers, Appl. Phys. Lett. 81, 562 (2002).

${ }^{11}$ R. M. Reano, Y. P. Kong, H. Y. Low, L. Tan, F. Wang, S. W. Pang, and A. F. Yee, J. Vac. Sci. Technol. B 22, 3294 (2004).

${ }^{12}$ D. J. Gundlach, C. S. Kuo, C. D. Sheraw, J. A. Nichols, and T. N. Jackson, Proc. SPIE 4466, 54 (2001).

${ }^{13}$ L. Tan, Y. P. Kong, S. W. Pang, and A. F. Yee, J. Vac. Sci. Technol. B 22, 2486 (2004).

${ }^{14}$ W. H. Kim, A. J. Makinen, N. Nikolov, R. Shashidhar, H. Kim, and Z. H. Kafafi, Appl. Phys. Lett. 80, 3844 (2002).

${ }^{15}$ T. Granlund, T. Nyberg, L. S. Roman, M. Svensson, and O. Inganas, Adv. Mater. (Weinheim, Ger.) 12, 269 (2000).

${ }^{16}$ I. Kymissis, C. D. Dimitrakopoulos, and S. Purushothaman, J. Vac. Sci. Technol. B 20, 956 (2002).

${ }^{17}$ Z. Wang, J. Zhang, R. Xing, J. Yuan, D. Yan, and Y. Han, J. Am. Chem. Soc. 125, 15278 (2003).

${ }^{18}$ N. Koch, A. Elschner, J. Schwartz, and A. Kahn, Appl. Phys. Lett. 82, 2281 (2003). 